\title{
Spacetime Structure near Generic Horizons and Soft Hair
}

\author{
Daniel Grumiller@, ${ }^{1,2,3,{ }^{*}}$ Alfredo Pérez $\oplus^{2, \dagger}$ M. M. Sheikh-Jabbari $\odot,{ }^{3,4, \hbar}$ Ricardo Troncoso ${ }^{2, \S}$ and Céline Zwikel ${ }^{1, \|}$ \\ ${ }^{1}$ Institute for Theoretical Physics, TU Wien, Wiedner Hauptstrasse 8-10/136, A-1040 Vienna, Austria \\ ${ }^{2}$ Centro de Estudios Científicos (CECs), Av. Arturo Prat 514, Valdivia, Chile \\ ${ }^{3}$ School of Physics, Inst. for Research in Fundamental Sciences (IPM), P.O. Box 19395-5531, Tehran, Iran \\ ${ }^{4}$ The Abdus Salam ICTP, Strada Costiera 11, 34151 Trieste, Italy
}

(Received 11 September 2019; published 28 January 2020)

\begin{abstract}
We explore the spacetime structure near nonextremal horizons in any spacetime dimension greater than two and discover a wealth of novel results: (i) Different boundary conditions are specified by a functional of the dynamical variables, describing inequivalent interactions at the horizon with a thermal bath. (ii) The near horizon algebra of a set of boundary conditions, labeled by a parameter $s$, is given by the semidirect sum of diffeomorphisms at the horizon with "spin-s supertranslations." For $s=1$ we obtain the first explicit near horizon realization of the Bondi-Metzner-Sachs algebra. (iii) For another choice, we find a nonlinear extension of the Heisenberg algebra, generalizing recent results in three spacetime dimensions. This algebra allows us to recover the aforementioned (linear) ones as composites. (iv) These examples allow us to equip not only black holes, but also cosmological horizons with soft hair. We also discuss implications of soft hair for black hole thermodynamics and entropy.
\end{abstract}

DOI: 10.1103/PhysRevLett.124.041601

Horizons are among the most remarkable entities in geometries with Lorentzian signature, appear in different contexts, and lead to rich phenomenology, see, e.g., Refs. [1-4] and references therein. Black holes, by their very definition, have horizons [5], which are in turn responsible for their peculiar classical properties that are reflected in observables such as x-ray spectra from accretion disks [6,7], the gravitational wave spectrum of black hole mergers [8-11], or their shadow, as observed recently by the Event Horizon Telescope [12]. Moreover, horizons are responsible for semiclassical properties, such as Hawking temperature and black hole evaporation [13] or the universal result for the Bekenstein-Hawking entropy [14]. Cosmological horizons also have thermal properties [15] and the very predictions of inflation [16-18] about power spectra of primordial perturbations is a result of horizon crossing of the quantum fluctuations [19-21]. There are observer dependent acceleration horizons that lead to Rindler quanta $[22,23]$ and the Unruh effect [24]. Horizons also feature prominently in analog black holes (dumb holes) [25,26].

Thermodynamical properties of horizons have led to the idea of deriving Einstein's equations (or generalizations thereof) from thermodynamics [27-29]. Understanding horizons and the associated microstates quantum mechanically is

Published by the American Physical Society under the terms of the Creative Commons Attribution 4.0 International license. Further distribution of this work must maintain attribution to the author(s) and the published article's title, journal citation, and DOI. Funded by SCOAP . an ongoing research endeavor where considerable progress has been made already [30-35], partly thanks to the holographic principle $[36,37]$ as manifested by the AdS/CFT correspondence [38-40].

Nevertheless, nonextremal horizons (i.e., horizons at finite temperature) remain largely mysterious [41-46]. No reliable and universal results for their microstates are known so far. Even in the simpler case of gravity in three spacetime dimensions, concrete microstate proposals such as horizon fluff $[47,48]$ require $a d$ hoc assumptions [49]. It is thus of considerable interest to understand nonextremal horizons as deeply as possible.

Another motivation for our work is to clarify possible relations between various different proposals for infinitedimensional near horizon symmetries [including symmetries resembling Bondi-Metzner-van der Burg-Sachs (BMS) [50,51], Virasoro and others], which may seemingly appear to be in conflict with each other, see, e.g., Refs. [52-56] and also Refs. [57-76].

In this work we derive generic properties of nonextremal horizons, assumed to be in equilibrium with a thermal bath, in any spacetime dimension greater than two. The physical properties of the thermal bath are modeled by the way we impose boundary conditions, and we shall describe various different well-motivated choices leading to infinitedimensional near horizon symmetries. We prove that they generically span soft hair excitations in the sense of Hawking, Perry, and Strominger [77] (see also Refs. [78-86]). While our methods are general, we focus on Einstein gravity (possibly with cosmological constant). 
We discuss in detail a wide class of boundary conditions, labeled by a parameter $s$, whose near horizon symmetry algebra is given by the semidirect sum of diffeomorphisms at the spacelike section of the horizon with spin-s supertranslations. In particular, the case of $s=1$ contains the first explicit near horizon realization of BMS in any dimension. Moreover, we also propose a special type of boundary conditions whose near horizon symmetries are described by a suitable nonlinear extension of the Heisenberg algebra, generalizing the results found in three spacetime dimensions $[53,87]$. This algebra allows us to recover the aforementioned ones as composites, in a way that we shall make precise.

Since our analysis is valid for any nonextremal horizon it applies in particular to generic Kerr black holes [88] (including Schwarzschild) and its generalizations that include NUT charge $[89,90]$. Some more detailed analysis may be found in Ref. [91].

Near horizon expansion.- - Let us consider a metric in $D \geq 3$ spacetime dimensions that features a nonextremal horizon (see Refs. [5,92-94] and references therein for various notions of horizon). Our working definition of a nonextremal horizon is that the line element can be brought into a Rindler-like form [22],

$$
d s^{2}=-\kappa^{2} \rho^{2} d t^{2}+d \rho^{2}+\Omega_{a b} d x^{a} d x^{b}+\ldots,
$$

where $\kappa$ is the surface gravity (with $\kappa \neq 0$ to guarantee nonextremality), $t$ is time, and $\rho$ is some radial coordinate that vanishes at the horizon. The "horizon metric," $\Omega_{a b}$ [where $a=1,2, \ldots(D-2)$ ], has nonvanishing determinant, $\Omega=\operatorname{det} \Omega_{a b} \neq 0$, to avoid degeneracy. The ellipsis denotes higher order terms or rotation or boost terms, which we spell out explicitly by defining a suitable near horizon expansion for the metric.

Our main assumption is that the horizon is free from singularities and the metric permits a Taylor expansion in $\rho$ in the near horizon region. Consistency with our assumptions above and with smoothness of the horizon determines the near horizon behavior of the metric, which in a corotating frame is given by [95]

$$
\begin{array}{ll}
g_{t t}=-\kappa^{2} \rho^{2}+\mathcal{O}\left(\rho^{3}\right), & g_{\rho \rho}=1+\mathcal{O}(\rho), \\
g_{t \rho}=\mathcal{O}\left(\rho^{2}\right), & g_{\rho a}=f_{\rho a} \rho+\mathcal{O}\left(\rho^{2}\right), \\
g_{t a}=f_{t a} \rho^{2}+\mathcal{O}\left(\rho^{3}\right), & g_{a b}=\hat{\Omega}_{a b}+\mathcal{O}\left(\rho^{2}\right),
\end{array}
$$

where $\hat{\Omega}_{a b}$ and $\Omega_{a b}$ are diffeomorphic to each other. All coefficients in Eq. (2) can depend on time $t$ and the transverse coordinates $x^{a}$, but not on the radius $\rho$.

The near horizon expansion (2) is preserved by a set of diffeomorphisms generated by vector fields $\xi^{\mu}$

$$
\xi^{t}=\frac{\eta}{\kappa}+\mathcal{O}(\rho), \quad \xi^{\rho}=\mathcal{O}\left(\rho^{2}\right), \quad \xi^{a}=\eta^{a}+\mathcal{O}\left(\rho^{2}\right),
$$

where $\eta^{a}$ depends arbitrarily on $x^{a}$, while $\eta$ depends additionally on $t$ subject to the condition $\partial_{t} \eta+\eta^{a} \partial_{a} \kappa=\delta \kappa$. The dynamical fields $\mathcal{P}$ and $\mathcal{J}_{a}$ defined by

$$
\mathcal{P}:=\frac{\sqrt{\Omega}}{8 \pi G}, \quad \mathcal{J}_{a}:=\frac{\sqrt{\Omega}}{16 \pi G \kappa}\left(\partial_{t} f_{\rho a}-2 f_{t a}\right),
$$

transform as

$$
\begin{gathered}
\delta \mathcal{P}=\eta^{a} \partial_{a} \mathcal{P}+\mathcal{P} \partial_{a} \eta^{a}, \\
\delta \mathcal{J}_{a}=\mathcal{P} \partial_{a} \eta+\eta^{c} \partial_{c} \mathcal{J}_{a}+\mathcal{J}_{c} \partial_{a} \eta^{c}+\mathcal{J}_{a} \partial_{c} \eta^{c} .
\end{gathered}
$$

The near horizon symmetries are spanned by generators, co-dimension two surface charges, that can be calculated using different methods; see, e.g., Refs. [96-98]. Their variations,

$$
\delta Q\left[\eta, \eta^{a}\right]=\int d^{D-2} x\left[\eta \delta \mathcal{P}+\eta^{a} \delta \mathcal{J}_{a}\right]
$$

turn out to be nontrivial and finite. To unveil the near horizon symmetries as a next step we impose boundary conditions that lead to integrable charges.

Fixing boundary conditions.-To obtain the charges $Q\left[\eta, \eta^{a}\right]$ (rather than their variations) we need precise boundary conditions by specifying the allowed variations of the metric. To admit the most general boundary conditions we rewrite the near horizon expansion (2) in a rotating frame, implemented through an Arnowitt-DeserMisner decomposition [99]. Metric components $g_{t \mu}$ (with $\mu=t, \rho, a)$ determine lapse function $N$ and shift vector $N^{i}=\left(N^{\rho}, N^{a}\right)$, which are Lagrange multipliers in the canonical formulation. Their leading order boundary behavior determines the sources (in holographic literature) or, equivalently, the chemical potentials (in black hole thermodynamics literature). In a rotating frame, lapse and shift expand as

$N=\mathcal{N} \rho+\mathcal{O}\left(\rho^{2}\right), \quad N^{a}=\mathcal{N}^{a}+\mathcal{O}\left(\rho^{2}\right), \quad N^{\rho}=\mathcal{O}\left(\rho^{2}\right)$.

The corotating falloff is recovered for $\mathcal{N}^{a}=0, \mathcal{N}=\kappa$.

Chemical potentials $\mathcal{N}$ and $\mathcal{N}^{a}$ appear in the boundary term $I_{B}$ that has to be added to the bulk Hamiltonian action for a well-defined variation principle [96]. It is evaluated in the limit of small $\rho$ and its variation reads

$$
\delta I_{B}=-\int d t d^{D-2} x\left(\mathcal{N} \delta \mathcal{P}+\mathcal{N}^{a} \delta \mathcal{J}_{a}\right),
$$

where $\mathcal{J}_{a}$ acquires additional terms as compared to Eq. (4) in a rotating frame [100]. Following Ref. [101], integrability of the boundary term (8) generically requires 


$$
\mathcal{N}=\frac{\delta F}{\delta \mathcal{P}}, \quad \mathcal{N}^{a}=\frac{\delta F}{\delta \mathcal{J}_{a}}
$$

for some functional $F\left[\mathcal{P}, \mathcal{J}_{a}\right]=\int d^{D-2} x \mathcal{F}\left(\mathcal{P}, \mathcal{J}_{a}\right)$. Therefore, the boundary conditions are fully fixed only once this functional is specified.

As spacetime does not possess a boundary near the horizon, different choices for the chemical potentials (9) amount to different prescriptions for the black hole interactions with a thermal bath, without the need of specifying the microscopic structure of the surrounding atmosphere. Thus, we are following an old and standard practice, like in electromagnetism of different media: all of the relevant information about the microscopic structure of the material is summarized in Maxwell's theory through the precise way one fixes the chemical potential at the boundary of the body, e.g., by fixing the time component of the gauge potential $A_{t}$ for a conductor (Dirichlet), or its normal derivative for a dielectric medium (Neumann).

Compatibility of time evolution with near horizon symmetries implies that the symmetry generator parameters in Eq. (6) acquire the same field dependence as the chemical potentials in Eq. (9), with $\mathcal{N} \rightarrow \eta$ and $\mathcal{N}^{a} \rightarrow \eta^{a}$. Thus, integrability of the boundary term (8) implies integrability of the symmetry generators (6).

We focus next on special choices for $\mathcal{F}\left(\mathcal{P}, \mathcal{J}_{a}\right)$ that lead to different sets of boundary conditions with an infinite number of near horizon symmetries.

BMS-like symmetries.-Retaining the full set of diffeomorphisms along the spacelike section of the horizon leads to an infinite amount of near horizon symmetries. This is achieved, e.g., by fixing $\delta \mathcal{N}^{a}=0$, while the lapse $\mathcal{N}$ can still be allowed to depend on $\mathcal{P}$. We propose a family of boundary conditions labeled by a parameter $s$,

$$
\mathcal{F}\left(\mathcal{P}, \mathcal{J}_{a}\right)=\mathcal{N}_{(s)} \frac{\mathcal{P}^{r+1}}{r+1}+\mathcal{N}^{a} \mathcal{J}_{a}
$$

where $\mathcal{N}_{(s)}$ is fixed, $\delta \mathcal{N}_{(s)}=0$, and $r=s /(D-2)$. This choice, together with Eq. (9), implies

$$
\mathcal{N}=\mathcal{N}_{(s)} \mathcal{P}^{r} \Rightarrow \eta=\eta_{(s)} \mathcal{P}^{r}
$$

The variation of the charges (6) integrates as

$$
Q\left[\eta_{(s)}, \eta^{a}\right]=\int d^{D-2} x\left[\eta_{(s)} \mathcal{P}_{(s)}+\eta^{a} \mathcal{J}_{a}\right]
$$

with $\mathcal{P}_{(s)}=\mathcal{P}^{r+1} /(r+1)$. Hence, from Eq. (5), the transformation law of the fields is given by

$$
\begin{aligned}
\delta \mathcal{P}_{(s)}= & \eta^{a} \partial_{a} \mathcal{P}_{(s)}+(r+1) \mathcal{P}_{(s)} \partial_{a} \eta^{a}, \\
\delta \mathcal{J}_{a}= & (r+1) \mathcal{P}_{(s)} \partial_{a} \eta_{(s)}+r \eta_{(s)} \partial_{a} \mathcal{P}_{(s)} \\
& +\eta^{c} \partial_{c} \mathcal{J}_{a}+\mathcal{J}_{a} \partial_{c} \eta^{c}+\mathcal{J}_{c} \partial_{a} \eta^{c},
\end{aligned}
$$

so that the near horizon Poisson bracket algebra reads

$$
\begin{aligned}
\left\{\mathcal{J}_{a}(x), \mathcal{P}_{(s)}(y)\right\} & =\left(r \mathcal{P}_{(s)}(y) \frac{\partial}{\partial x^{a}}-\mathcal{P}_{(s)}(x) \frac{\partial}{\partial y^{a}}\right) \delta(x-y), \\
\left\{\mathcal{P}_{(s)}(x), \mathcal{P}_{(s)}(y)\right\} & =0 \\
\left\{\mathcal{J}_{a}(x), \mathcal{J}_{b}(y)\right\} & =\left(\mathcal{J}_{a}(y) \frac{\partial}{\partial x^{b}}-\mathcal{J}_{b}(x) \frac{\partial}{\partial y^{a}}\right) \delta(x-y) .
\end{aligned}
$$

The algebra (14) is the semidirect sum of diffeomorphisms at the $(D-2)$-dimensional spacelike section of the horizon, generated by $\mathcal{J}_{a}(x)$, and a generalization of supertranslations spanned by $\mathcal{P}_{(s)}(x)$.

If the spacelike section of the horizon has the topology of $S^{D-2}$, the largest finite subalgebra is given by the semidirect sum of $s o(D-1,1)$ (Lorentz) and "spin-s translations," spanned by suitable subsets of $\mathcal{J}_{a}(x)$ and $\mathcal{P}_{(s)}(x)$, respectively.

For $D=3$, the algebra (14) for $s=0$ agrees with the one found in Refs. [52,69], while for $s=1$ it is the $\mathrm{BMS}_{3}$ algebra $[102,103]$. For generic $s$ it is the $W(0,-s)$ algebra $[104,105]$, where the supertranslations are generators with conformal weight $h=s+1$.

For $D \geq 4$, if the horizon metric is restricted to be conformal to the round sphere, $\Omega_{a b}=\Phi^{2} \Omega_{a b}^{S^{D-2}}$, the parameters $\eta^{a}$ reduce to the conformal Killing vectors of $S^{D-2}$, so that its associated generators $\mathcal{J}_{a}$ exactly span the Lorentz algebra $s o(D-1,1)$. It is then worth highlighting that our boundary conditions for $s=1$ provide the first explicit realization of the $\mathrm{BMS}_{D}$ algebra as near horizon symmetries in four and higher dimensions. Further aspects of $\mathrm{BMS}_{D}$ and its higher spin extensions may be found in Ref. [91].

For $s=0$, the algebra carries "scalar supertranslations" and agrees with the result in Refs. [52,69] for $D=3,4$.

Heisenberg-like symmetries.-The parameters $\eta^{a}$ in Eq. (6) are vectors, while their corresponding charges are 1-form densities of weight one. It is natural to swap their role, in the sense that the charges are 1-forms and their corresponding parameters become densities. This set of boundary conditions is obtained by choosing

$$
\mathcal{F}\left(\mathcal{P}, \mathcal{J}_{a}\right)=\mathcal{N}_{H} \mathcal{P}+\mathcal{N}_{H}^{a} \mathcal{J}_{a} \mathcal{P}^{-1},
$$

with $\mathcal{N}_{H}$ and $\mathcal{N}_{H}^{a}$ fixed $\left(\delta \mathcal{N}_{H}=0, \delta \mathcal{N}_{H}^{a}=0\right)$. The chemical potentials follow from Eq. (9), so that the symmetry parameters in Eq. (6) are now given by

$$
\eta^{a}=\eta_{H}^{a} \mathcal{P}^{-1}, \quad \eta=\eta_{H}-\eta_{H}^{a} \mathcal{J}_{a} \mathcal{P}^{-2} .
$$

The variation of the generators (6) then integrates as

$$
Q_{H}\left[\eta_{H}, \eta_{H}^{a}\right]=\int d^{D-2} x\left[\eta_{H} \mathcal{P}+\eta_{H}^{a} \mathcal{J}_{a}^{H}\right]
$$


with the 1 -form $\mathcal{J}_{a}^{H}:=\mathcal{J}_{a} \mathcal{P}^{-1}$. The transformation laws (5) now yields

$$
\begin{aligned}
\delta \mathcal{P} & =\partial_{a} \eta_{H}^{a}, \\
\delta \mathcal{J}_{a}^{H} & =\partial_{a} \eta_{H}-\eta_{H}^{b} F_{a b} \mathcal{P}^{-1},
\end{aligned}
$$

where $F_{a b}:=\partial_{a} \mathcal{J}_{b}^{H}-\partial_{b} \mathcal{J}_{a}^{H}$. The transformation laws (18) establish the Poisson bracket algebra

$$
\begin{aligned}
\left\{\mathcal{J}_{a}^{H}(x), \mathcal{P}(y)\right\} & =\frac{\partial}{\partial x^{a}} \delta(x-y), \\
\{\mathcal{P}(x), \mathcal{P}(y)\} & =0, \\
\left\{\mathcal{J}_{a}^{H}(x), \mathcal{J}_{b}^{H}(y)\right\} & =\mathcal{P}^{-1}(x) F_{b a}(x) \delta(x-y) .
\end{aligned}
$$

We note that Eq. (19a) implies $\left\{\mathcal{P}(x), F_{a b}(y)\right\}=0$.

In three dimensions $F_{a b}$ identically vanishes, so that the boundary conditions reduce to those in Refs. [53,87], which accommodate "soft hairy" black hole solutions, while Eq. (19) becomes equivalent to two copies of $\hat{u}(1)$ current algebras.

In $D>3$ dimensions the phase space restricted to configurations with $F_{a b}=0$ is preserved under the full set of asymptotic symmetries in Eq. (18). Examples are toroidal Kerr-AdS black holes and Schwarzschild black holes, together with their soft hair excitations. In these cases the 1 -form $\mathcal{J}_{a}^{H}$ is locally exact, $\mathcal{J}_{a}^{H}=: 8 \pi G \partial_{a} \mathcal{Q}$. Then, the only nonvanishing Poisson bracket (19a),

$$
\{\mathcal{Q}(x), \mathcal{P}(y)\}=\frac{1}{8 \pi G} \delta(x-y),
$$

yields the Heisenberg algebra. Quantizing the Poisson bracket algebra (20) (viz., replacing $i\{$,$\} by commutators$ [,]) yields canonical commutation relations with the factor $1 /(4 G)$ playing the role of Planck's constant $h$ [106]. This simple result could be relevant in semiclassical descriptions of black holes [107].

Compositeness, soft hair, and thermodynamics.-We discussed two classes of algebras, the BMS-like ones (14) in which the right-hand side (rhs) of commutators are linear in the generators $\left(\mathcal{J}_{a}, \mathcal{P}_{(s)}\right)$ and the Heisenberglike algebra spanned by $\left(\mathcal{J}_{a}^{H}, \mathcal{P}\right)$, in which the rhs of Eq. (19c) are nonlinear in generators. Remarkably, generators of the BMS-like algebras (14) emerge as composites in terms of generators of the Heisenberg-like algebra (19), $\mathcal{J}_{a}=\mathcal{J}_{a}^{H} \mathcal{P}$, and $\mathcal{P}_{(s)}=\mathcal{P}^{r+1} /(r+1)$. In this sense, the Heisenberg-like generators $\left(\mathcal{J}_{a}^{H}, \mathcal{P}\right)$ are the building blocks. We have thus generalized this feature observed first in three dimensions [87] to arbitrary dimensions. Note that in $D \geq 4$ the nonlinearity of the Heisenberg-like algebra is the key to establish the map between the generators.
All boundary conditions discussed here allow soft hair excitations in the sense of Ref. [77], i.e., gravitational excitations that carry no energy, but nonetheless are not pure gauge. For the BMS-like boundary conditions, the near horizon total Hamiltonian, given by the generator of unit time translations, in a nonrotating frame reads $H_{(s)}=Q\left[\partial_{t}\right]=$ $\int d^{D-2} x \mathcal{N}_{(s)} \mathcal{P}_{(s)}$. The spin-s supertranslations $\mathcal{P}_{(s)}$ correspond to soft hair charges since they commute with $H_{(s)}$, see Eq. (14). For Heisenberg-like boundary conditions, the generators $\mathcal{P}$ also stand for soft hair charges, since they commute with the near horizon Hamiltonian, which in a nonrotating frame reads $H_{H}=Q\left[\partial_{t}\right]=\int d^{D-2} x \mathcal{N}_{H} \mathcal{P}$.

We address now the Bekenstein-Hawking entropy. For BMS-like boundary conditions it reads $S=A /(4 G)=$ $2 \pi(r+1)^{[(1 /(r+1)]} \int d^{D-2} x\left(\mathcal{P}_{(s)}\right)^{[(1 /(r+1)]}$. Although soft hair excitations do not contribute to the energy, for $s \neq 0$ they contribute to the entropy through the modes of $\mathcal{P}_{(s)}$. Only for $s=0$ or for the Heisenberg-like boundary conditions soft hair excitations do not contribute to the BekensteinHawking entropy, which is given by the zero mode of $\mathcal{P}_{0}=\mathcal{P}[52,53]$,

$$
S=2 \pi \mathcal{P}_{0},
$$

with $\mathcal{P}_{0}=\int d^{D-2} x \mathcal{P}$.

Different choices of boundary conditions generically describe inequivalent thermodynamic ensembles. The chemical potentials correspond to the variables that are kept fixed. Demanding smoothness of the metric around the horizon implies that the lapse and shift are given by $\mathcal{N}=$ $\kappa=2 \pi \beta^{-1}$ and $\mathcal{N}^{a}=0$, where inverse temperature $\beta$ is the Euclidean time period. For the BMS-like boundary conditions the chemical potentials (11) are fixed as $\mathcal{N}_{(s)}=$ $(2 \pi / \beta)\left[(r+1) \mathcal{P}_{(s)}\right]^{-[r /(r+1)]}$ and thus, the first law is fulfilled as expected $\delta S=\beta \delta H_{(s)}$, where the variation of the total Hamiltonian includes the internal energy, as well as work terms. For the Heisenberg-like boundary conditions, the chemical potentials are given by $\mathcal{N}_{H}=2 \pi \beta^{-1}$, $\mathcal{N}_{H}^{a}=0$ and the first law reads $\delta S=\beta \delta H_{H}$. Note that in the latter case, as well as for $s=0$, temperature $T=\beta^{-1}$ is state independent.

Kerr black hole example.-Our results can be applied to arbitrary nonextremal black holes in diverse dimensions, for instance, black holes with cosmological constant or NUT charge. Here we give the essentials for the most interesting black hole, nonextremal Kerr [88]. Since the BMS-like generators are composites of the Heisenberg-like ones, it is enough to perform the analysis in the latter case. For this choice we have mixed boundary conditions that describe how the Kerr black hole interacts with a thermal bath: the metric component $g_{t t}$ is fixed, the metric component $g_{t \rho}$ is irrelevant, and the metric components $g_{t a}$ are allowed to fluctuate in a state-dependent way. Translated to Maxwell's theory these boundary conditions mean that the 
black hole horizon behaves like a conductor only with respect to $g_{t t}$, but not with respect to $g_{t a}$. The near horizon Heisenberg-like generators for Kerr black holes with event or inner horizon radii $r_{ \pm}$are given by $(\theta \in[0, \pi)$, $\varphi \sim \varphi+2 \pi)$,

$$
\begin{aligned}
\mathcal{P} & =\frac{r_{+}\left(r_{+}+r_{-}\right)}{8 \pi G} \sin \theta, \\
\mathcal{J}_{a}^{H} & =\delta_{a}^{\varphi} r_{-} \frac{r_{-}\left(r_{-}-r_{+}\right) \cos ^{2} \theta-r_{+}\left(3 r_{+}+r_{-}\right)}{2 \sqrt{r_{+} r_{-}}\left(r_{+}+r_{-} \cos ^{2} \theta\right)^{2}} \sin ^{2} \theta .
\end{aligned}
$$

$\mathcal{P}$ only has a monopole contribution (in the sense that it is proportional to the two-dimensional volume factor $\sin \theta$ ), while $\mathcal{J}_{a}^{H}$ only have a coexact part, $\mathcal{J}_{a}^{H}=\varepsilon_{a}{ }^{b} \partial_{b} \psi$, where $\psi=2 \arctan U+\left[\left(r_{+}-r_{-}\right) /\left(r_{+}+r_{-}\right)\right] U$ with $U=$ $\sqrt{r_{-} / r_{+}} \cos \theta$. The field strength defined below (18b) is nonzero, $F_{a b} \neq 0$, but the flux integrated over the horizon vanishes, $\int_{H} F=0$. Further details and a generalization to Kerr black holes with NUT charges (which introduces fluxes $\int_{H} F \neq 0$ ) can be found in Ref. [91].

Comments and further developments.-In the present work we focused on nonextremal black hole horizons. Our results also apply to cosmological horizons. Indeed, $\rho=0$ in Eq. (2) could be the observer horizon associated with a cosmological de Sitter patch. It is worthwhile to apply our analysis to cosmology and study possible consequences for cosmic perturbation theory and hence cosmological observables with soft hair. Our near horizon charges could be relevant in the derivation of cosmological consistency relations [110,111] or for the infinite set of Ward identities of the adiabatic modes [112].

An interesting generalization is to include matter or gravitational wave fluxes through the horizon, allowing a wider class of configurations, including nonstationary black holes. Such an analysis may pave the way to address Hawking radiation and the information paradox.

While we focused on matterless Einstein gravity, results from three dimensions [53,87,113-119] suggest that the BMS-like and Heisenberg-like algebras are universal and apply also to general relativity with matter, higher derivative theories, and possibly other modifications of Einstein gravity. It could be rewarding to verify (or falsify) this claim by considering such examples.

We are grateful to Hamid Afshar, Oscar Fuentealba, Dario Francia, Hernán González, Wout Merbis, Jorge Noreña, Emilio Ojeda, Miguel Pino, Pablo Rodríguez, David Tempo, Raphaela Wutte, and Hossein Yavartanoo for collaboration on aspects of near horizon symmetries. D. G. was supported by the Austrian Science Fund (FWF), Projects No. P 28751 and No. P 30822. M. M. Sh-J. would like to thank the hospitality of ICTP HECAP where this work finished and acknowledges the support by INSF Grant No. 950124 and Saramadan Grant No. ISEF/M/ 98204. C. Z. was supported by the Austrian Science Fund
(FWF), Projects No. P 30822 and No. M 2665. D. G. and M. M. Sh-J. acknowledge the Iran-Austria IMPULSE project grant, supported and run by Khawrizmi University. D. G., A.P., and R. T. acknowledge travel support from the Conicyt Grant No. REDES170052. We also thank the hospitality of the Erwin Schrödinger Institute (ESI) in Vienna during the program "Higher Spins and Holography," as well as the organizers of the "Second Hermann Minkowski Meeting on the Foundations of Spacetime Physics" in Albena, where part of this work was completed. This research has been partially supported by Fondecyt Grants No. 1161311, No. 1171162, No. 1181031, and No. 1181496. The Centro de Estudios Científicos (CECs) is funded by the Chilean Government through the Centers of Excellence Base Financing Program of Conicyt.

*grumil@ hep.itp.tuwien.ac.at

†aperez@cecs.cl

*jabbari@theory.ipm.ac.ir

\$troncoso@cecs.cl

"zwikel@hep.itp.tuwien.ac.at

[1] J. M. Bardeen, B. Carter, and S. W. Hawking, Commun. Math. Phys. 31, 161 (1973).

[2] R. Bousso, Rev. Mod. Phys. 74, 825 (2002).

[3] T. Padmanabhan, Phys. Rep. 406, 49 (2005).

[4] A. Ashtekar and B. Krishnan, Living Rev. Relativity 7, 10 (2004).

[5] S. Hawking and G. Ellis, The Large Scale Structure of Space-Time (Cambridge University Press, Cambridge, England, 1973).

[6] N. I. Shakura and R. A. Sunyaev, Astron. Astrophys. 24, 337 (1973).

[7] R. A. Remillard and J. E. McClintock, Annu. Rev. Astron. Astrophys. 44, 49 (2006).

[8] L. Blanchet, T. Damour, B. R. Iyer, C. M. Will, and A. G. Wiseman, Phys. Rev. Lett. 74, 3515 (1995).

[9] F. Pretorius, Phys. Rev. Lett. 95, 121101 (2005).

[10] L. Blanchet, Living Rev. Relativity 9, 4 (2006).

[11] B. P. Abbott et al. (Virgo and LIGO Scientific Collaborations), Phys. Rev. Lett. 116, 061102 (2016).

[12] K. Akiyama et al. (EHT Collaboration), Astrophys. J. Lett. 875, L1 (2019).

[13] S. W. Hawking, Commun. Math. Phys. 43, 199 (1975).

[14] J. D. Bekenstein, Phys. Rev. D 7, 2333 (1973).

[15] G. W. Gibbons and S. W. Hawking, Phys. Rev. D 15, 2738 (1977).

[16] A. A. Starobinsky, Pis'ma Zh. Eksp. Teor. Fiz. 30, 719 (1979) [JETP Lett. 30, 682 (1979)].

[17] A. H. Guth, Phys. Rev. D 23, 347 (1981).

[18] A. D. Linde, Phys. Lett. 108B, 389 (1982).

[19] V. F. Mukhanov and G. V. Chibisov, Pis'ma Zh. Eksp. Teor. Fiz. 33, 549 (1981) [JETP Lett. 33, 532 (1981)].

[20] A. H. Guth and S. Y. Pi, Phys. Rev. Lett. 49, 1110 (1982).

[21] V. F. Mukhanov, H. A. Feldman, and R. H. Brandenberger, Phys. Rep. 215, 203 (1992).

[22] W. Rindler, Am. J. Phys. 34, 1174 (1966). 
[23] N. D. Birrell and P. C. W. Davies, Quantum Fields in Curved Space (Cambridge University Press, Cambridge, England, 1982).

[24] W. G. Unruh, Phys. Rev. D 14, 870 (1976).

[25] W. G. Unruh, Phys. Rev. Lett. 46, 1351 (1981).

[26] C. Barcelo, S. Liberati, and M. Visser, Living Rev. Relativity 8, 12 (2005).

[27] T. Jacobson, Phys. Rev. Lett. 75, 1260 (1995).

[28] T. Padmanabhan, Rep. Prog. Phys. 73, 046901 (2010).

[29] E. P. Verlinde, J. High Energy Phys. 04 (2011) 029.

[30] A. Strominger and C. Vafa, Phys. Lett. B 379, 99 (1996).

[31] A. Strominger, J. High Energy Phys. 02 (1998) 009.

[32] M. Guica, T. Hartman, W. Song, and A. Strominger, Phys. Rev. D 80, 124008 (2009).

[33] M. Van Raamsdonk, Gen. Relativ. Gravit. 42, 2323 (2010); Int. J. Mod. Phys. D 19, 2429 (2010).

[34] J. Maldacena and L. Susskind, Fortschr. Phys. 61, 781 (2013).

[35] D. Harlow, Rev. Mod. Phys. 88, 015002 (2016).

[36] G. 't Hooft, Salamfestschrift (World Scientific, Singapore, 1993); Conf. Proc. C930308, 284 (1993).

[37] L. Susskind, J. Math. Phys. (N.Y.) 36, 6377 (1995).

[38] J. M. Maldacena, Adv. Theor. Math. Phys. 2, 231 (1998).

[39] S. S. Gubser, I. R. Klebanov, and A. M. Polyakov, Phys. Lett. B 428, 105 (1998).

[40] E. Witten, Adv. Theor. Math. Phys. 2, 253 (1998).

[41] S. W. Hawking, Phys. Rev. D 14, 2460 (1976).

[42] J. Preskill, in International Symposium on Black Holes, Membranes, Wormholes and Superstrings Woodlands, Texas, 1992 (World Scientific, Houston, Texas, 1992), pp. 22-39.

[43] S. D. Mathur, Fortschr. Phys. 53, 793 (2005).

[44] K. Skenderis and M. Taylor, Phys. Rep. 467, 117 (2008).

[45] A. Almheiri, D. Marolf, J. Polchinski, and J. Sully, J. High Energy Phys. 02 (2013) 062.

[46] K. Papadodimas and S. Raju, Phys. Rev. Lett. 112, 051301 (2014).

[47] H. Afshar, D. Grumiller, and M. M. Sheikh-Jabbari, Phys. Rev. D 96, 084032 (2017).

[48] M. M. Sheikh-Jabbari and H. Yavartanoo, Phys. Rev. D 95, 044007 (2017).

[49] H. Afshar, D. Grumiller, M. M. Sheikh-Jabbari, and H. Yavartanoo, J. High Energy Phys. 08 (2017) 087.

[50] H. Bondi, M. van der Burg, and A. Metzner, Proc. R. Soc. A 269, 21 (1962).

[51] R. Sachs, Phys. Rev. 128, 2851 (1962).

[52] L. Donnay, G. Giribet, H. A. González, and M. Pino, Phys. Rev. Lett. 116, 091101 (2016).

[53] H. Afshar, S. Detournay, D. Grumiller, W. Merbis, A. Perez, D. Tempo, and R. Troncoso, Phys. Rev. D 93, 101503(R) (2016).

[54] S. Carlip, Phys. Rev. Lett. 120, 101301 (2018).

[55] R. F. Penna, J. High Energy Phys. 10 (2017) 049.

[56] S. Haco, S. W. Hawking, M. J. Perry, and A. Strominger, J. High Energy Phys. 12 (2018) 098.

[57] G. 't Hooft, Nucl. Phys. B335, 138 (1990).

[58] G. 't Hooft, Phys. Scr. T36, 247 (1991).

[59] L. Susskind, L. Thorlacius, and J. Uglum, Phys. Rev. D 48, 3743 (1993).
[60] S. Carlip, Phys. Rev. D 51, 632 (1995).

[61] A. P. Balachandran, L. Chandar, and A. Momen, Nucl. Phys. B461, 581 (1996).

[62] S. Carlip, in Field Theory, Integrable Systems and Symmetries (Les Publications CRM: Montreal, Canada, 1997).

[63] A. Ashtekar, J. Baez, A. Corichi, and K. Krasnov, Phys. Rev. Lett. 80, 904 (1998).

[64] S. Carlip, Phys. Rev. Lett. 82, 2828 (1999).

[65] M. Hotta, K. Sasaki, and T. Sasaki, Classical Quantum Gravity 18, 1823 (2001).

[66] G. 't Hooft, Int. J. Mod. Phys. D 15, 1587 (2006).

[67] B. R. Majhi and T. Padmanabhan, Phys. Rev. D 86, 101501(R) (2012).

[68] H. Afshar, S. Detournay, D. Grumiller, and B. Oblak, J. High Energy Phys. 03 (2016) 187.

[69] L. Donnay, G. Giribet, H. A. González, and M. Pino, J. High Energy Phys. 09 (2016) 100.

[70] D. Lust, Fortschr. Phys. 66, 1800001 (2018).

[71] V. Chandrasekaran, É. É. Flanagan, and K. Prabhu, J. High Energy Phys. 11 (2018) 125.

[72] R. F. Penna, arXiv:1812.05643.

[73] S. Haco, M. J. Perry, and A. Strominger, arXiv:1902.02247.

[74] L. Donnay and G. Giribet, Classical Quantum Gravity 36, 165005 (2019).

[75] L. Donnay and C. Marteau, Classical Quantum Gravity 36, 165002 (2019).

[76] V. Chandrasekaran and K. Prabhu, J. High Energy Phys. 10 (2019) 229.

[77] S. W. Hawking, M. J. Perry, and A. Strominger, Phys. Rev. Lett. 116, 231301 (2016).

[78] A. Averin, G. Dvali, C. Gomez, and D. Lust, J. High Energy Phys. 06 (2016) 088.

[79] G. Compère and J. Long, Classical Quantum Gravity 33, 195001 (2016).

[80] M. Mirbabayi and M. Porrati, Phys. Rev. Lett. 117, 211301 (2016).

[81] S. W. Hawking, M. J. Perry, and A. Strominger, J. High Energy Phys. 05 (2017) 161.

[82] D. Carney, L. Chaurette, D. Neuenfeld, and G. W. Semenoff, Phys. Rev. Lett. 119, 180502 (2017).

[83] R. Bousso and M. Porrati, Classical Quantum Gravity 34, 204001 (2017).

[84] W. Donnelly and S. B. Giddings, Phys. Rev. D 96, 086013 (2017).

[85] Y. Angelopoulos, S. Aretakis, and D. Gajic, Phys. Rev. Lett. 121, 131102 (2018).

[86] G. Raposo, P. Pani, and R. Emparan, Phys. Rev. D 99, 104050 (2019).

[87] H. Afshar, D. Grumiller, W. Merbis, A. Perez, D. Tempo, and R. Troncoso, Phys. Rev. D 95, 106005 (2017).

[88] R. P. Kerr, Phys. Rev. Lett. 11, 237 (1963).

[89] A. H. Taub, Ann. Math. 53, 472 (1951).

[90] E. Newman, L. Tamubrino, and T. Unti, J. Math. Phys. (N.Y.) 4, 915 (1963).

[91] D. Grumiller, A. Pérez, M. M. Sheikh-Jabbari, R. Troncoso, and C. Zwikel, arXiv:1908.09833.

[92] S. A. Hayward, Phys. Rev. D 49, 6467 (1994).

[93] A. Ashtekar, C. Beetle, O. Dreyer, S. Fairhurst, B. Krishnan, J. Lewandowski, and J. Wiśniewski, Phys. Rev. Lett. 85, 3564 (2000). 
[94] A. Ashtekar and B. Krishnan, Phys. Rev. Lett. 89, 261101 (2002).

[95] Additional terms omitted in Eq. (2) are either pure gauge or inconsistent with integrability of boundary charges or refer to a rotating frame. Nonintegrable charges would be needed if we were interested in dynamical situations where gravitational waves (or matter) have a net flux through the horizon. In our current work we exclude this possibility, while allowing for cases with equal in and out fluxes through the horizon, like the case of large AdS black holes which are in equilibrium with their own Hawking radiation.

[96] T. Regge and C. Teitelboim, Ann. Phys. (N.Y.) 88, 286 (1974).

[97] R. M. Wald and A. Zoupas, Phys. Rev. D 61, 084027 (2000).

[98] G. Barnich and F. Brandt, Nucl. Phys. B633, 3 (2002).

[99] R. L. Arnowitt, S. Deser, and C. W. Misner, Gen. Relativ. Gravit. 40, 1997 (2008).

[100] In general, $\mathcal{J}_{a}=16 \pi G \Omega_{a b} \pi_{(0)}^{\rho b}$, where $\pi_{(0)}^{\rho b}$ stands for the leading term of the canonical momenta conjugate to $g_{\rho b}$.

[101] A. Pérez, D. Tempo, and R. Troncoso, J. High Energy Phys. 06 (2016) 103.

[102] A. Ashtekar, J. Bicak, and B. G. Schmidt, Phys. Rev. D 55, 669 (1997).

[103] G. Barnich and G. Compere, Classical Quantum Gravity 24, F15 (2007).

[104] S. Gao, C. Jiang, and Y. Pei, Communications in algebra 39, 397 (2011).

[105] A. Farahmand Parsa, H. R. Safari, and M. M. SheikhJabbari, J. High Energy Phys. 03 (2019) 143.
[106] D. Grumiller and M. M. Sheikh-Jabbari, Int. J. Mod. Phys. D 27, 1847006 (2018).

[107] Symmetry generating diffeomorphisms that lead to the Heisenberg algebra (20) consist of $\mathcal{P}$ current and area preserving shear deformations of the horizon. The latter are crucial in the membrane paradigm [108,109]. Our analysis can provide the setting to formulate and quantize the membrane paradigm [106].

[108] K. S. Thorne, R. Price, and D. Macdonald, Black Holes: The Membrane Paradigm, edited by K. S. Thorne (Yale University Press, New Haven, Connecticut, 1986).

[109] R. H. Price and K. S. Thorne, Phys. Rev. D 33, 915 (1986).

[110] K. Hinterbichler, L. Hui, and J. Khoury, J. Cosmol. Astropart. Phys. 08 (2012) 017.

[111] A. Joyce, J. Khoury, and M. Simonović, J. Cosmol. Astropart. Phys. 01 (2015) 012.

[112] K. Hinterbichler, L. Hui, and J. Khoury, J. Cosmol. Astropart. Phys. 01 (2014) 039.

[113] M. R. Setare and H. Adami, Phys. Lett. B 760, 411 (2016).

[114] D. Grumiller, A. Perez, S. Prohazka, D. Tempo, and R. Troncoso, J. High Energy Phys. 10 (2016) 119.

[115] M. R. Setare and H. Adami, Nucl. Phys. B914, 220 (2017).

[116] M. Ammon, D. Grumiller, S. Prohazka, M. Riegler, and R. Wutte, J. High Energy Phys. 05 (2017) 031.

[117] D. Grumiller, A. Perez, D. Tempo, and R. Troncoso, J. High Energy Phys. 08 (2017) 107.

[118] D. Grumiller, P. Hacker, and W. Merbis, J. High Energy Phys. 02 (2018) 010.

[119] M. R. Setare and H. Adami, arXiv:1711.08344. 